\title{
An opportunity to improve exercise test accuracy by incorporating high frequency QRS (HFQRS) waveform assessment
}

\author{
Gregory S. Thomas, MD, MPH, MASNC, ${ }^{\mathrm{a}, \mathrm{b}}$ and Roxana Ghashghaei, MD ${ }^{\mathrm{a}, \mathrm{b}}$ \\ a MemorialCare Heart \& Vascular Institute, Southern California, CA \\ b Division of Cardiology, University of California, Irvine School of Medicine, Orange, CA
}

Received Jan 3, 2019; accepted Jan 4, 2019

doi: $10.1007 / \mathrm{s} 12350-019-01600-7$

\section{See related article, pp. 2063-2075}

Exercise testing has served as the workhorse for the evaluation of ischemia for over half a century. Once electrocardiography was added to exercise testing, ${ }^{1}$ ECG changes occurring during ventricular repolarization, as manifested by ST depression, became the standard for an abnormal ECG response. Over time, test accuracy increased with the incorporation of the degree and time of onset of ST duration, duration of ST depression during recovery, exercise-induced symptoms, exercise duration, blood pressure response, heart rate recovery, and development of ventricular arrhythmia. ${ }^{2}$

In 1989, Detrano and colleagues estimated the sensitivity and specificity of exercise testing to be $68 \%$ and $77 \%$, respectfully. ${ }^{3}$ This was based on a metaanalysis of 147 studies performed during the previous 22 years. The addition of adjunctive imaging with myocardial perfusion imaging (MPI) or echocardiography to exercise testing increased sensitivity another $\approx 10-20 \%$ with $\mathrm{a} \approx 10 \%$ increase in specificity. $^{4}$

However, in most developed countries today, patients present with less severe CAD than when these earlier studies were carried out. ${ }^{2,5,6}$ As it is harder for a test to detect disease with a test if the disease is less severe, ${ }^{2,7,8}$ the sensitivity and specificity of exercise testing have likely suffered since the meta-analysis of Detrano. This has resulted in clinicians increasingly

Reprint requests: Gregory S. Thomas, MD, MPH, MASNC, MemorialCare Heart \& Vascular Institute, Southern California, CA; gthomas1@memorialcare.org

J Nucl Cardiol 2020;27:2076-9.

$1071-3581 / \$ 34.00$

Copyright $(\subset) 2019$ American Society of Nuclear Cardiology. relying on adjunctive imaging to obtain greater test accuracy. As well, patients presenting for stress testing are often older than in earlier times and less likely to be able to achieve $\geq 85 \%$ of maximum predicted heart rate. Each limits exercise testing accuracy, again encouraging adjunctive imaging.

One option to increase exercise test sensitivity in this time of decreasing CAD severity is to expand the threshold for a positive test to include not only $\geq 1 \mathrm{~mm}$ of flat or horizontal ST depression, but also $>1.5 \mathrm{~mm}$ of upsloping ST depression. ${ }^{2}$ Doukky and colleagues have also recently suggested the potential of lowering the ECG threshold for abnormal ST depression during vasodilator stress to $\geq 0.5 \mathrm{~mm}$ of flat or horizontal ST depression. ${ }^{9,10}$

In this issue of the Journal, Balfour, Bourque, and colleagues at the University of Virginia explore a new opportunity to improve exercise test sensitivity by quantifying the subtle changes that occur in the propagation of the wavefront of depolarization as it moves through areas of ischemic myocardium. ${ }^{11}$ Building on the work of earlier investigators, these authors found that incorporating an assessment of these high frequency, low amplitude signals that occur during the QRS complex to standard $1 \mathrm{~mm}$ of ST depression criteria increased test sensitivity to detect ischemia as assessed by MPI, improved the prediction of outcomes over a 5.3 years of follow-up and added to the prediction of patients who would be referred for early revascularization.

\section{HOW IS ISCHEMIA EVALUATED DURING VENTRICULAR DEPOLARIZATION?}

The standard ECG uses filtering to assess waveforms with frequencies of $0.05-100 \mathrm{~Hz}$. These waveforms have amplitudes measured in mVolts $(\mathrm{mV})$. Note, a typical $\mathrm{R}$ wave measures $5-15 \mathrm{mV}$ and will be 
measured as 5-15 mm on a standard ECG tracing. These waveforms are termed low frequency, high amplitude. However, the converse, high frequency, low amplitude waveforms also occur during the ventricular depolarization. As reviewed by Goldberger and colleagues, these high frequency waveforms make up $<3 \%$ of QRS amplitude. These waveforms, abbreviated as HFQRS waveforms are approximately 100 times smaller in amplitude and are thus measured in microvolts $(\mu \mathrm{V})$ rather than millivolts. ${ }^{12}$ Investigators have long considered the diagnostic potential of these potentials on the resting ECG.

HFQRS waveforms can be measured if an ECG is obtained at a high sampling rate, e.g., 1000 samples per second $(1000 \mathrm{~Hz})$, also called an expanded bandwidth, and if a filter is used to minimize signals other than high frequency waveforms of 150-250 Hz.

In 1987, Abboud and colleagues demonstrated a decrease in HFQRS waveform amplitude during ischemia induced by balloon inflation in a coronary artery. ${ }^{13}$ They averaged multiple QRS complexes to decrease extraneous electrical noise and applied a filter to suppress waveforms not in the $150-250 \mathrm{~Hz}$ range. The intensity of the HFQRS waveforms was expressed by using their root-mean-square (RMS) values which reflect the average amplitude of the signal, be it positive or negative. The RMS is calculated by squaring the amplitude of each electrical signal, determining the mean of these squares and then the taking the square root of this mean.

In 2010, Ringborn, Pettersson and colleagues also assessed HFQRS during balloon inflation. ${ }^{14,15}$ They used software developed by the Signal Processing Group at Lund University, Sweden. The onset and offset of the QRS were determined from a signal averaged ECG in the standard frequency range (0.05$100 \mathrm{~Hz}$ ) prior to the next step of using a filter to measure the electrical potentials in the $150-250 \mathrm{~Hz}$ range. They found the degree of decrease in HFQRS amplitude during balloon inflation, which they assessed by RMS, served as a better arbiter of ischemia, as assessed by Tc-99 $\mathrm{m}$ sestamibi injection during balloon inflation, than ST elevation occurring on the standard ECG.

\section{WHAT IS THE MECHANISM FOR THE DECREASE IN HFQRS WAVEFORM INTENSITY DURING ISCHEMIA?}

The currently accepted mechanism as to why HFQRS waveform intensity decreases during ischemia was reviewed by Amit and colleagues. ${ }^{16}$ The extensively branching His-Purkinje system results in a vast array of myocardial activation sites during normal depolarization resulting in a depolarization wavefront with numerous HFQRS signals. However, ischemia results in a slowing of the conduction from one myocyte to another, resulting in a decrease in the amplitude of high frequency waveforms as depolarization moves through this area of ischemia. This is illustrated in Figure 2 in the article by Balfour et al. ${ }^{11}$

In 2012, Sharir and colleagues evaluated HFQRS during exercise MPI in 996 patients. ${ }^{17}$ HFQRS was assessed using advanced signal processing software (HyperQ) developed by Biological Signal Processing (Tel Aviv, Israel). Criteria for HFQRS positivity in an individual ECG lead was a decrease in the RMS of HFQRS waveforms measured during the QRS of $\geq 50 \%$ from their maximum to their minimum with a minimum absolute $\geq 1 \mu \mathrm{V}$ decrease. Using ROC analysis, they found the optimal criteria for test accuracy in an individual patient was 3 or more leads with an abnormal HFQRS response. HFQRS outperformed ST depression as a predictor of MPI detected ischemia and the number of positive HFQRS leads correlated with ischemic extent. An example of a patient with severe ischemia on MPI with 9 ECG leads with an abnormal HFQRS response is shown in the Figure 1.

Rosenmann and colleagues evaluated the addition of HFQRS to ST segment evaluation during exercise testing in 113 women. ${ }^{18}$ Using coronary angiography as the gold standard, the addition of HFQRS improved specificity. In addition, the number of leads with abnormal HFQRS response correlated with the degree of angiographic CAD. Conti and co-workers found the addition of HFQRS to ST segment depression in 622 patients undergoing stand-alone exercise testing improved the prediction of cardiac events over the ensuing 6 months. ${ }^{19}$ Lipton and colleagues had earlier found HFQRS to add to ST depression in the prediction of ischemia in 139 patients undergoing exercise MPI. ${ }^{20}$ HyperQ software was used in each of these studies.

The outstanding contribution of Balfour, Bourque, and colleagues adds to previous investigations in their findings that HFQRS prospectively provided prognostic information above and beyond ST depression over a prolonged period of follow-up, 5.3 years, and their use of an overt strategy to add HFQRS to ST depression rather than to consider the two measures competitive. ${ }^{11}$ In the setting of decreasing CAD severity, this inclusive strategy is welcome. Using both ST depression and $\geq 3$ leads with abnormal HFQRS as their criteria for positivity, they also found HFQRS to result in a net reclassification of $25 \%$ of patients studied.

As this was a study of patients referred for exercise MPI, it disadvantaged the comparison of the accuracy of ST depression relative to HFQRS waveforms. Some of the patients undergoing an evaluation of CAD by stress 
A
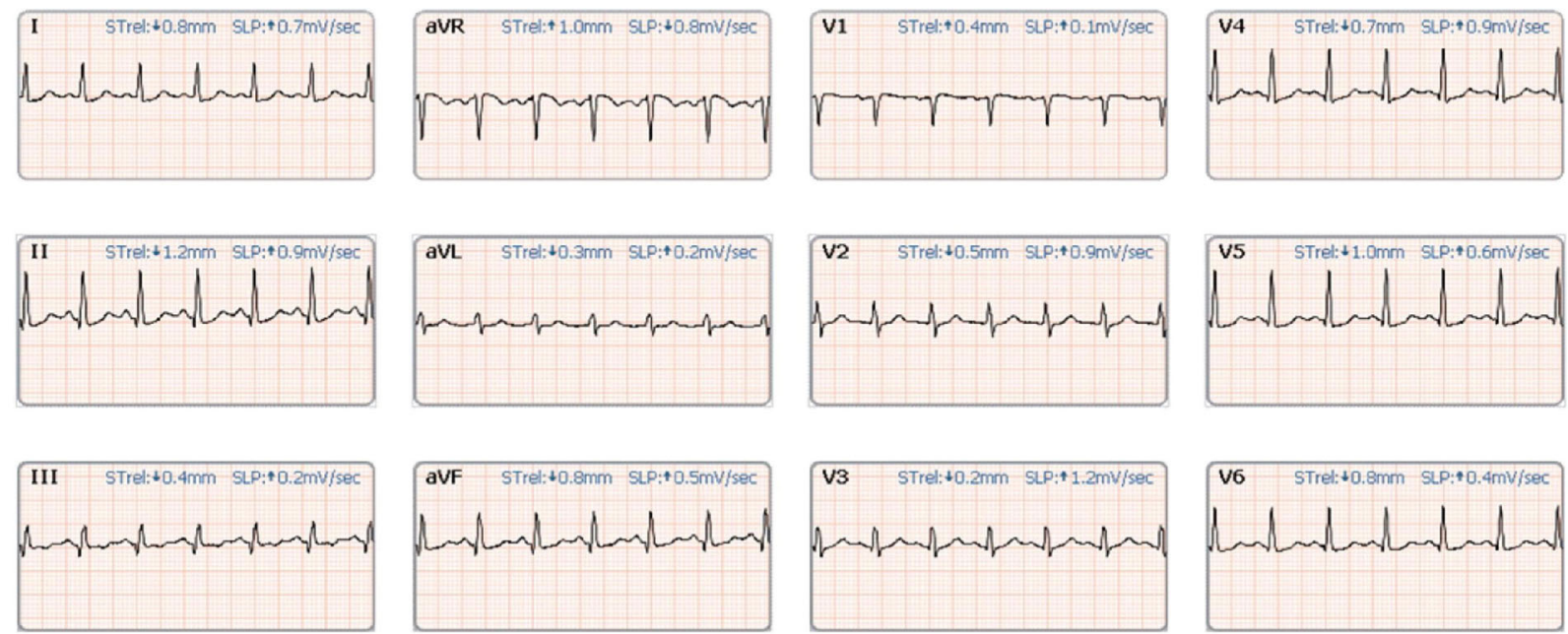

$25 \mathrm{~mm} / \mathrm{sec} 10 \mathrm{~mm} / \mathrm{mV} \quad$ Filters: Baseline $50 \mathrm{~Hz}$ Multicycle
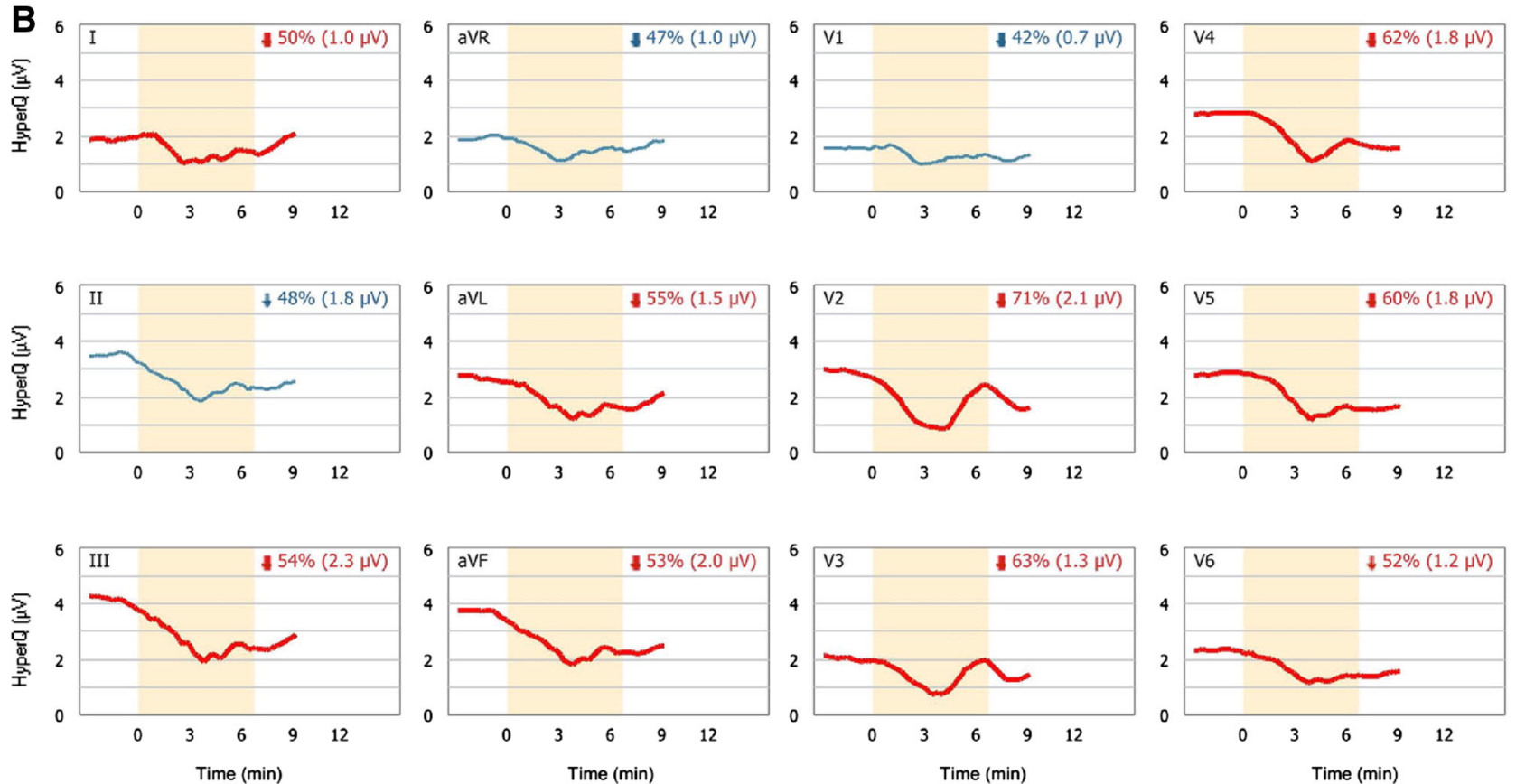

Figure 1. An example of a patient with severe ischemia on myocardial perfusion imaging with exercise-induced decreases in high frequency QRS waveform amplitude, as measured by root mean square, in multiple leads (A) associated with mild ST depression by ECG (B). HFQRS decreases $>50 \%$ are signified by red trend lines. HyperQ on the $x$ axis signifies the decrease in the HFQRS root mean square. Reprinted with permission from ${ }^{17}$.

testing would undergone stand-alone exercise testing at which time they may have been found to be positive by ST depression. Hence, such patients would have not proceeded to exercise MPI and not have been included in this study.

Based on their study, Balfour and colleagues also raise the potential that adjunctive MPI could be avoided in patients whose exercise test demonstrates both a normal ST and HFQRS response, and who presumably do not develop exercise-induced symptoms consistent with ischemia.

\section{LIMITATIONS}

The chief limitations of HFQRS are two-fold: (1) A lack of availability of software to assess HFQRS on most ECG equipment used for stress testing, though the software and high sampling rate are available on some 
Schiller equipment. (2) The need for sinus rhythm with a relatively noise-free QRS which is $<120 \mathrm{~ms}$ in duration. In the study of Balfour, $27 \%$ of patients were excluded for these electrocardiographic reasons.

Future work that will build confidence in HFQRS technology and add further diagnostic and prognostic information are multi-center studies. Almost all studies to date have been single center. Additionally, studies to date have also included patients who achieve $>85 \%$ of maximum predicted heart rate. Just as ST depression adds important information in patients whose exercise is submaximal, HFQRS may as well, suggesting that this population be studied.

\section{CONCLUSIONS}

Practitioners and investigators with access to this software technology are encouraged to consider the addition of HFQRS in electrocardiographic assessment during exercise testing, stand-alone, or with adjunctive imaging, and further determine the role it will play in the diagnostic and prognostic evaluation of patients with suspected CAD. Vendors are encouraged to build this technology into new stress testing equipment and to create the opportunity to incorporate it into existing equipment.

\section{Disclosure}

Gregory S. Thomas acknowledges royalties from Ellestad's stress testing: Principles and practice, Oxford University Press, 2018 and serving on the Speakers bureau of Astellas Pharma. Roxana Ghashghaei has no disclosures.

\section{References}

1. Wood FC, Wolferth CC. Angina pectoris: the clinical and electrocardiographic phenomena of the attack and their comparison with the effects of experimental temporary coronary occlusion. Arch Intern Med 1931;47(3):339-65.

2. Thomas GS, Wann LS, Ellestad M. Ellestad's stress testing: principles and practice. 6th ed. New York: Oxford University Press; 2018.

3. Detrano R, Gianrossi R, Mulvihill D, et al. Exercise-induced ST segment depression in the diagnosis of multivessel coronary disease: a meta analysis. J Am Coll Cardiol 1989;14(6):1501-8.

4. Fihn SD, Gardin JM, Abrams J, et al. 2012 ACCF/AHA/ACP/ AATS/PCNA/SCAI/STS guideline for the diagnosis and management of patients with stable ischemic heart disease. J Am Coll Cardiol 2012;60:e44-164.

5. Jouni H, Askew JW, Crusan DJ, Miller TD, Gibbons RJ. Temporal trends of single- photon emission computed tomography myocardial perfusion imaging in patients with coronary artery disease: a 22-year experience from a tertiary academic medical center. Circ Cardiovasc Imaging 2017;10:e005628.

6. Duvall WL, Rai M, Ahlberg AW, O'Sullivan DM, Henzlova MJ. A multi-center assessment of the temporal trends in myocardial perfusion imaging. J Nucl Cardiol 2015;22(3):539-51.

7. Brenner H, Gefeller O. Variation of sensitivity, specificity, likelihood ratios and predictive values with disease prevalence. Stat Med 1997;16(9):981-91.

8. Wong HB, Lim GH. Measures of diagnostic accuracy: sensitivity, specificity, PPV and NPV. Proc Singap Healthc 2011;20(4):316-8.

9. Doukky R, Nigatu A, Khan R, et al. Prognostic significance of ischemic electrocardiographic changes with regadenoson stress myocardial perfusion imaging. J Nucl Cardiol 2018. https://doi. org/10.1007/s12350-018-1415-4.

10. Thomas G, Medina C. Examining a novel threshold for defining electrocardiographic ischemia with vasodilator stress. J Nucl Cardiol 2018. https://doi.org/10.1007/s12350-018-01491-0.

11. Balfour PC, Gonzalez JA, Shaw PW, et al. High-frequency QRS analysis to supplement ST evaluation in exercise stress electrocardiography: incremental diagnostic accuracy and net reclassification. J Nucl Cardiol 2018. https://doi.org/10.1007/s12 350-018-01530-w.

12. Goldberger AL, Bhargava V, Froelicher V, Covell J. Effect of myocardial infarction on high-frequency QRS potentials. Circulation 1981;64(1):34-42.

13. Abboud S, Cohen RJ, Selwyn A, Ganz P, Sadeh D, Friedman PL. Detection of transient myocardial ischemia by computer analysis of standard and signal-averaged high-frequency electrocardiograms in patients undergoing percutaneous transluminal coronary angioplasty. Circulation 1987;76(3):585-96.

14. Ringborn M, Pettersson J, Persson E, et al. Comparison of highfrequency QRS components and ST-segment elevation to detect and quantify acute myocardial ischemia. J Electrocardiol 2010;43(2):113-20.

15. Pettersson J, Pahlm O, Carro E, et al. Changes in high-frequency QRS components are more sensitive than ST-segment deviation for detecting acute coronary artery occlusion. J Am Coll Cardiol 2000;36(6): 1827-34.

16. Amit G, Granot Y, Abboud S. Quantifying QRS changes during myocardial ischemia: insights from high frequency electrocardiography. J Electrocardiol 2014;47(4):505-11.

17. Sharir T, Merzon K, Kruchin I, et al. Use of electrocardiographic depolarization abnormalities for detection of stress-induced ischemia as defined by myocardial perfusion imaging. Am J Cardiol 2012;109(5):642-50.

18. Rosenmann D, Mogilevski Y, Amit G, Davrath LR, Tzivoni D. High-frequency QRS analysis improves the specificity of exercise ECG testing in women referred for angiography. J Electrocardiol 2013;46(1):19-26.

19. Conti A, Bianchi S, Grifoni C, et al. High-frequency QRS analysis superior to conventional ST-segment analysis of women with chest pain. Am J Emerg Med 2016;34(3):437-42.

20. Lipton JA, Warren SG, Broce M, et al. High-frequency QRS electrocardiogram analysis during exercise stress testing for detecting ischemia. Int J Cardiol 2008;124(2):198-203.

Publisher's Note Springer Nature remains neutral with regard to jurisdictional claims in published maps and institutional affiliations. 